\title{
Non-Local MRI Upsampling
}

\author{
José V. Manjón ${ }^{a}$, Pierrick Coupé ${ }^{b}$, Antonio Buades ${ }^{c, d}$, \\ Vladimir Fonov ${ }^{\mathrm{b}}$, D. Louis Collins ${ }^{\mathrm{b}}$, Montserrat Robles ${ }^{\mathrm{a}}$
}

a Instituto de Aplicaciones de las Tecnologías de la Información y de las Comunicaciones Avanzadas (ITACA), Universidad Politécnica de Valencia, Camino de Vera s/n, 46022 Valencia, Spain.

b McConnell Brain Imaging Centre, Montreal Neurological Institute, McGill University, Montreal, Canada.

${ }^{c}$ Université Paris Descartes, 45 rue des Saints Pères, 75270 Paris Cedex 06, France.

d Dpt Matemàtiques i Informàtica, Universitat Illes Balears, Ctra Valldemossa km 7.5, 07122 Palma de Mallorca, Spain.

*Corresponding author. José V. Manjón

Instituto de Aplicaciones de las Tecnologías de la Información y de las Comunicaciones Avanzadas (ITACA), Universidad Politécnica de Valencia, Camino de Vera s/n, 46022 Valencia, Spain.

Tel.: (+34) 963877000 Ext. 75275 Fax: (+34) 963879009.

E-mail address: jmanjon@fis.upv.es 


\begin{abstract}
In Magnetic Resonance Imaging image resolution comes limited by several factors such as hardware or time limitations. In many cases, the acquired images has to be upsampled to match on any specific resolution, in such cases, image interpolation techniques has been traditionally applied. However, traditional interpolation techniques are not able to recover high frequency information of the underlying high resolution data. In this paper, a new reconstruction method is proposed to recover some of this high frequency information by using a data adaptive patch-based regularization in combination with a subsampling coherence constraint. The proposed method has been evaluated on synthetic and real clinical cases and compared with traditional interpolation methods. The proposed method has shown to outperform classical interpolation methods compared.
\end{abstract}

Keywords: MRI, interpolation, superresolution.

\title{
Abbreviations:
}

NLM: Non Local Means

MNLM3D: Multiresolution Non Local Means 3D

SNR: Signal to Noise Ratio

PSNR: Peak Signal to Noise Ratio

LR: Low Resolution

HR: High Resolution

SR: SuperResolution

DTI: Diffusion Tensor Imaging

fMRI: functional Magnetic Resonance Imaging 


\section{Introduction}

In medical imaging, the image voxel size comes limited by a number of factors such as imaging hardware, Signal to Noise Ratio (SNR), time limitations or patient's comfort. In many cases, the acquired voxel size has to be decreased to fit with a concrete resolution requirement. In such situations, interpolation techniques have been traditionally used (Thévenaz et al., 2000; Lehmann et al., 1999).

Common interpolation techniques, like linear interpolation or spline-based methods, have been extensively used in the past to decrease voxel size and increase apparent data resolution due to its simplicity and low computational burden. These techniques assume that the existing points in the Low Resolution (LR) image may be represented using some kind of generic functions $\left(\mathrm{n}^{\text {th }}\right.$ order polynomials in case of B-Splines for example) with additional assumption on smoothness (i.e avoiding discontinuities on $1^{\text {st }}$ order derivatives), followed by calculating the values of the points in High Resolution (HR) image using found coefficients. However, such techniques invent new points assuming that the existing ones (in the LR images) have the same value in the HR images which is only valid at homogeneous regions. As a result, interpolated images are typically blurred versions of its corresponding HR reference images.

Only recently, SuperResolution (SR) techniques have emerged as an alternative to increase effectively the resolution of the reconstructed data (Carmi et al., 2006). In MRI, superresolution techniques have been previously applied to increase image resolution on functional MRI (fMRI) (Kornprobst et al, 2003) and Diffusion Tensor Imaging (DTI) studies (Peled and Yeshurun, 2001). Unfortunately, most of these techniques are based on the acquisition of multiple LR images with small shifts which is time consuming and therefore not adequate for typical clinical settings.

As an alternative to multiple image SR techniques, single image SR techniques perform resolution enhancement using the expected degradation model (Zhang and Cham, 2008) or exploiting the normal pattern redundancy available on 
image data (Ebrahimi and Vrscay, 2008; Xin Li, 2008; Protter et al., 2009; Elad and Datsenko, 2009). These later have recently shown very competitive results compared to classical interpolation methods. As for denoising, where the NLmeans demonstrated high abilities to denoise images (Buades et al., 2005), these patch-based interpolation techniques enable to significantly improve the classical results.

The exemplar-based or patch-based methods take advantage of the selfsimilarity of the image. In fact, instead of acquiring several images of the same object to increase the image resolution, the patch-based approaches consider that is possible to extract similar information by using the information redundancy within a single image. The patch-based methods can be viewed as fractal approaches at the same scale (Ebrahimi and Vrscay, 2008).

As described in Ebrahimi and Vrscay (2008) and deeply detailed in Elad and Datsenko (2009), the examples/patches can be involved in a different way during image interpolation. The patches can be used to learn some priors on the image in to order to improve regularization procedures (Roth and Black, 2005). The patches can also be used directly to reconstruct the image as in inpainting or texture synthesis (Criminisi, 2004, Efros et al, 1999). More recently, hybrid methods using examples within regularization framework have been proposed (Ebrahimi and Vrscay, 2008; Elad and Datsenko, 2009; Xin Li, 2008) and demonstrate very high qualities.

The method proposed in this paper shares some characteristics with single image SR techniques based on self-similarity using a regularization expression (Ebrahimi and Vrscay, 2008; Xin Li, 2008; Protter et al., 2009). However, by taking into account the specificities of the MR acquisition, the proposed method is based on different regularity and coherence assumptions typically met in MR imaging. Moreover, a robust coarse to fine scheme is involved to achieve the reconstruction procedure. An extensive validation on synthetic and real MR datasets is proposed to demonstrate the ability of our proposed method to increase voxel resolution in MR data. 


\section{Material and Methods}

In MR imaging, voxels in LR data $y$ can be related to the corresponding $x \mathrm{HR}$ voxels using this expression:

$$
y_{p}=\frac{1}{N} \sum_{i=1}^{N} x_{i}+n
$$

where $y_{p}$ is the observed LR voxel at location $p, x_{i}$ is each one of the $N \mathrm{HR}$ voxels contained within this LR voxel and $n$ is some additive noise from the measurement process. This model assumes that LR voxels can be well modeled as the average of the corresponding HR voxel values. Moreover, the Rician noise typically present in magnitude images can be well-approximated within the imaged object as Gaussian distributed for typical clinical SNR values (SNR>3) (Nowak, 1999; Gudbjartsson and Patz 1995).

The aim of any reconstruction/interpolation method is to find the $x_{i}$ values from the $y_{j}$ values which is a very ill-posed problem since there are infinite $x_{i}$ values that meet such condition.

In classical interpolation techniques such as linear interpolation the $x_{i}$ values are calculated as a weighted average of the LR voxels $y$.

$$
x_{i}=y_{j} \quad \text { if }(\mathrm{i}=\mathrm{j}) \text { and } x_{i}=\frac{1}{M} \sum_{i=1}^{M} w_{i j} y_{j} \text { otherwise }
$$

where the weights $w$ are calculated as function of the Euclidean distance between the coordinates of the new voxels and the LR surrounding $M$ existing ones. As can be noted, none of these methods takes into account the fact that existing $L R$ voxels are the average of the underlying HR voxels. 


\section{Proposed method}

The method proposed in this paper is rooted on the application of a 3D variant of the Non-Local Means filter. The use of this method enforces the structure preserving rather than imposing any smoothness constraint. Besides, if the presence of noise is minimized on the LR image by applying an appropriate filter it can be imposed as a new constraint that the downsampled version of the reconstructed image $\hat{x}$ has to be exactly the same as the original LR image $y$ for all location $p$. Therefore, the used fidelity constraint can be written as:

$$
y_{p}-\frac{1}{N} \sum_{i=1}^{N} \widehat{x}_{i}=0 \quad \forall p \in y
$$

This constraint has been previously applied in the SR context and referred as subsampling consistency (Banerjee and Jawahar, 2008). Due to the presence of noise, equation 3 cannot be directly used. To simplify the problem, the LR data is first denoised using a recently proposed robust denoising method (MNLM3D) for 3D MR images (Coupé et al., 2008b) based in the well known Non-local Means filter early proposed by Buades et al. (2005). It has been demostrated that such filter is able to remove noise effectively while affecting minimally the image structure. For low SNR MR data, where the Gaussian noise approximation is no longer valid, some Rician adapted denoising methods (Manjón et al., 2008; Wiest-Daessle et al., 2008) can be used.

Finally, the proposed method uses as input data a pre-interpolated version of the LR filtered data. It is an iterative procedure based on the following two steps:

\section{1) Patch-based non-local Regularization}

To perform high quality image upsampling, a patch-based non-local regularization procedure is proposed. As shown in (Xi Lin, 2008, Protter et al., 2009), the non-local means algorithm can be efficiently adapted to regularization tasks. In fact, voxels with similar local neighborhoods can be 
considered as similar and averaged to recover local image structures. In our approach, we apply a patch-based regularization by using a voxel-wise 3D version of the NLM filter (Coupé et al., 2008a) with a preselection step adapted to the progressive reconstruction used.

$$
\begin{aligned}
& \hat{x}_{p}^{t+1}=\frac{1}{C_{p}} \sum_{\forall q \in \Omega} w\left(\hat{x}_{p}^{t}, \hat{x}_{q}^{t}\right) \hat{x}_{q}^{t} \\
& w\left(\hat{x}_{p}^{t}, \hat{x}_{q}^{t}\right)=e^{-\frac{\mid N\left(\hat{x}_{p}^{t}\right)-N\left(\hat{x}_{q}^{t}\right)^{2}}{h^{2}}}
\end{aligned}
$$

Where $\hat{x}_{p}^{t}$ is each voxel of the actual image at iteration $t, N\left(\hat{x}_{p}^{t}\right)$ and $N\left(\hat{x}_{q}^{t}\right)$ are image 3D patches around voxels $p$ and $q, \Omega$ is a local search area surrounding the voxel being processed and $C$ is a normalization constant. For further details see Coupe et al. (2008a).

In our proposed non-local regularization, the preselection is based on the Mean Absolute Difference (MAD) of the mean values of compared patches. Patches with MAD smaller than $3 h / \sqrt{N}$ were used in the averaging, where $h$ is a filtering parameter related to the degree of smoothing and $N$ is the number of voxels used in the averaging. This approach allows to perform a preselection based on this $h$ parameter instead of patch local moments as suggested by Coupé et al. (2008a) which is better suited for our coarse to fine regularization purposes.

$$
w\left(\hat{x}_{p}^{t}, \hat{x}_{q}^{t}\right)=\left\{\begin{array}{rr}
e^{-\frac{\left|N\left(\hat{x}_{p}^{t}\right)-N\left(\hat{x}_{p}^{t}\right)\right|^{2}}{h^{2}}} \text { if } & \left|\mu_{p}^{t}-\mu_{q}^{t}\right|<3 h / \sqrt{N} \\
0 & \text { otherwise. }
\end{array}\right.
$$

Where $\mu_{p}^{t}$ is the mean value of a 3D patch centered at voxel $p$ at iteration $t$.

By this way, the voxel under study is reconstructed using all similar voxels (in the patch distance sense) in the image or in a restricted search area. Thus, the natural redundancy of information contained in the image is used to efficiently reconstruct the current voxel. Contrary to classical interpolation methods based 
on a straight-forward approach of modeling the data using only the local neighborhood of a voxel, the patch-based non-local regularization is able to use the context of a voxel to use only relevant information from the search area.

2) Mean correction: As shown in by Xu et al. (2008), the non-local means filter does not ensure the mean preservation of the reconstructed value. In our case, the mean preservation of every HR reconstructed area corresponding to a given LR voxel is a critical issue since the reconstruction of $\hat{x}$ image must be accomplished under the constraint described in equation 3 in order to be a physically plausible solution. Thus, we proposed a mean correction step in order to ensure that the reconstructed HR image will be consistent with the original LR one.

As the downsampled version of the reconstructed HR $\hat{x}$ image has to be equal to the original $L R$ image, the local mean value of the reconstructed HR voxels is corrected to fit with the value of the original LR voxel by adding the corresponding offset.

$$
\hat{x}=\hat{x}+N N(D(\hat{x})-y)
$$

where $D$ is a downsampling operator that transforms actual reconstructed $\mathrm{HR}$ data to the original LR and NN is a Nearest Neighbor interpolation operation that interpolates LR data to HR.

This two steps (Regularization-Correction) are iteratively repeated until no significative difference is found between two consecutive reconstructions (MAD inferior to a given tolerance). A block diagram of the proposed method can be observed in figure 1. 


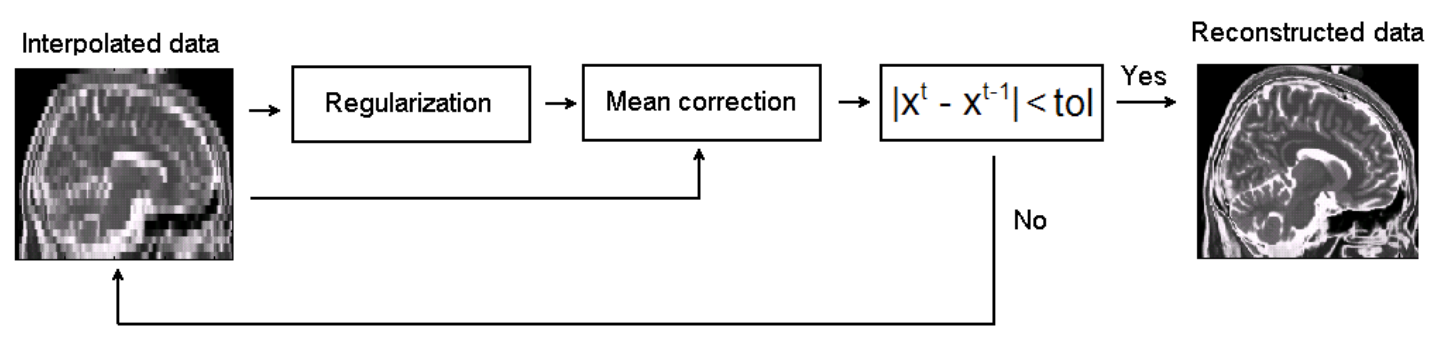

Figure 1. Block diagram of the proposed method.

\section{Experiments and results}

\subsection{Implementation details}

The proposed method requires an initial interpolation to start the reconstruction process. In our experiments, we used the $3^{\text {th }}$ order B-Spline interpolation as implemented in MATLAB 7.4 (Mathworks Inc.).

The described patch-based Non-local regularization scheme has 3 free parameters which are the radius of the search area $v$, the radius of the patch $f$ and the degree of filtering $h$. Parameters $v$ and $f$ were set to 3 and 1 as suggested in Coupé et al. (2008) for denoising purposes which represents $7 \times 7 \times 7$ and $3 \times 3 \times 3$ voxels 3D windows respectively.

To adjust $h$ parameter a decremental approach was used similar to the one used by Buades et al for Non-Local Demosaicing (Buades et al., 2009). A set of decreasing $h$ values were used until no significative difference were found in the results between consecutive $h$ values $(h=[\sigma / 2, \sigma / 4, \sigma / 8, \ldots])$. Each $h$ value was used until no significative differences were found between two consecutive reconstructions and then the value of $h$ is decreased to the half. This process is repeated until no significative differences among scales (values of $h$ ) are found. Here $\sigma$ represent the standard deviation of the imaged object $y$. In our experiments the tolerance was set to $0.002 \sigma$ which was found a good choice experimentally. In figure 2, an example of the evolution of Peak Signal to noise Ration (PSNR), and Mean Absolute Deviation (MAD) of the iterative process is presented to better show the way that the proposed method works. 

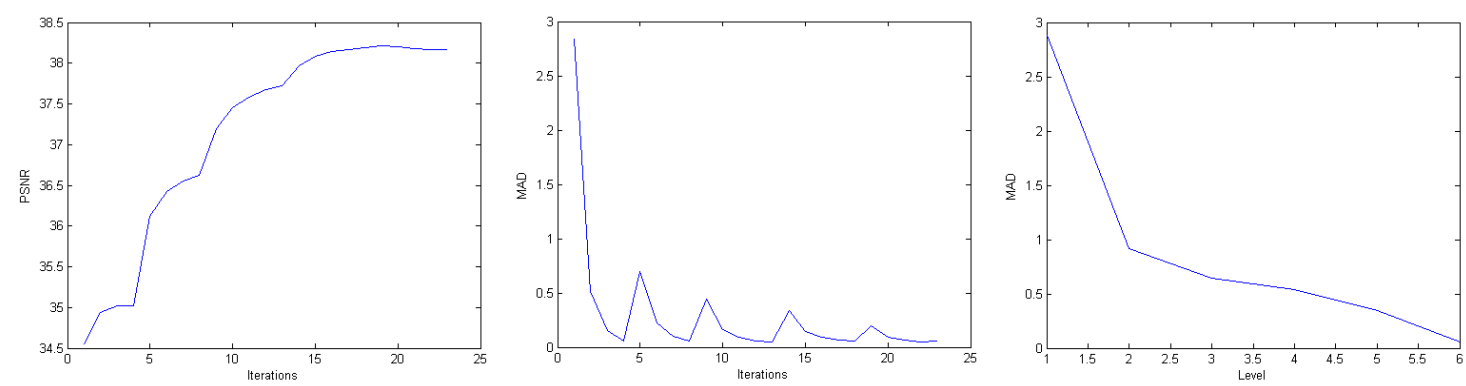

Figure 2. On the left: evolution of the PSNR in function of the iterations (the jumps in the PSNR values are due to the different values of $h$ ). In the center the MAD value between each two iterations is shown (for a given $h$ value). In the right the MAD in different scales ( $h$ values).

\subsection{Simulated data evaluation}

The first experiment consisted in reconstructing downsampled versions of a HR T1w volume of $181 \times 217 \times 180$ voxels (voxel resolution $1 \mathrm{~mm}^{3}$ ) that corresponds to HR T1-w Brainweb phantom (Cocosco et al.,1997). This HR volume was downsampled in the $z$ direction to simulate different slice thickness $(2,3,5,7$ and $9 \mathrm{~mm}$ ). These simulated LR data were then upsampled again to $1 \mathrm{~mm}^{3}$. The proposed method was compared to the Nearest Neighbor (NN), Trilinear, Cubic and B-Spline $\left(3^{\text {th }}\right.$ order) interpolation methods as implemented on MATLAB 7.4. (Mathworks Inc). In this first experiment no noise was added to simplify the analysis of the results (we wanted to evaluate the reconstruction process not the denoising process). Peak Signal to Noise Ratio (PSNR) metric was used to evaluate the performance of the different methods. The results can be observed on table 1 and figure 2 . As can be noticed the proposed method obtained the best results in all the cases. 
The same experiment was repeated but this time using noisy LR data to find out how the noise and the filtering process affects the results. The LR data was corrupted by adding a Gaussian distributed noise with an amplitude similar to that obtained on real clinical datasets ( $4 \%$ of maximum intensity). This noisy data was then filtered using the MNLM3D method. Finally, all the upsampling methods were applied to these filtered data. Results are shown in table 2 and figure 4. Again, the proposed method obtained the best results in all the cases.

Table 1. PSNR values of the different methods compared for several slices thicknesses.

\begin{tabular}{cccccc}
\hline Slice Thickness $(\mathbf{m m})$ & $\mathbf{2}$ & $\mathbf{3}$ & $\mathbf{5}$ & $\mathbf{7}$ & $\mathbf{9}$ \\
\hline NN & 31.33 & 27.59 & 23.93 & 22.13 & 20.85 \\
Linear & 34.49 & 30.17 & 25.27 & 23.07 & 21.55 \\
Cubic & 36.91 & 31.61 & 25.95 & 23.56 & 21.96 \\
B-Spline & 37.80 & 32.40 & 26.06 & 23.55 & 21.94 \\
Proposed & $\mathbf{4 1 . 2 8}$ & $\mathbf{3 5 . 4 2}$ & $\mathbf{2 8 . 4 4}$ & $\mathbf{2 4 . 9 0}$ & $\mathbf{2 2 . 6 6}$ \\
\hline
\end{tabular}

Table 2. PSNR values of the different methods compared for several slices thicknesses (noisy case, $4 \%)$.

\begin{tabular}{cccccc}
\hline Slice Thickness $\mathbf{( m m})$ & $\mathbf{2}$ & $\mathbf{3}$ & $\mathbf{5}$ & $\mathbf{7}$ & $\mathbf{9}$ \\
\hline NN & 29.81 & 26.82 & 23.57 & 21.89 & 20.68 \\
Linear & 31.51 & 28.64 & 24.67 & 22.71 & 21.31 \\
Cubic & 32.61 & 29.60 & 25.27 & 23.17 & 21.69 \\
B-Spline & 32.93 & 30.09 & 25.38 & 23.17 & 21.69 \\
Proposed & $\mathbf{3 4 . 5 4}$ & $\mathbf{3 2 . 2 1}$ & $\mathbf{2 7 . 9 5}$ & $\mathbf{2 5 . 0 8}$ & $\mathbf{2 2 . 9 9}$ \\
\hline
\end{tabular}




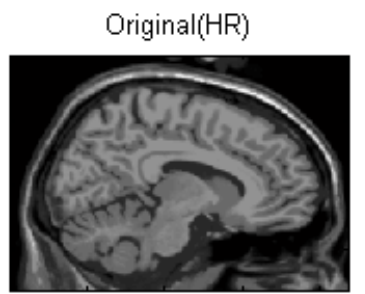

Original( $\mathrm{HR})$

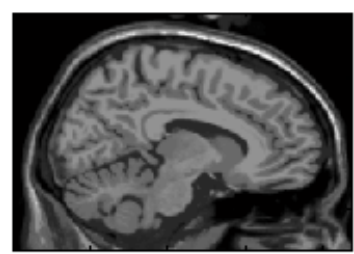

Original( $(\mathrm{HR})$

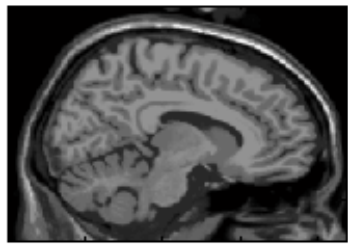

Original( $(\mathrm{HR})$

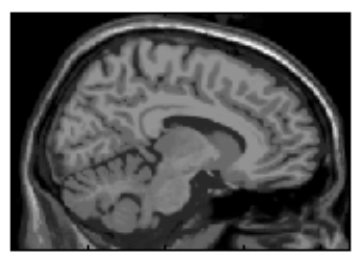

Original( $(\mathrm{HR})$

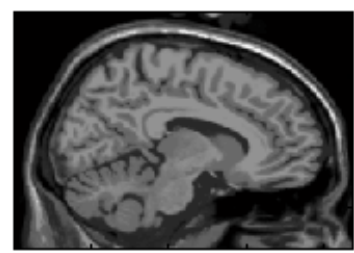

NN PSNR=31.32dB

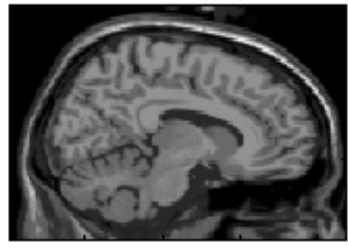

NN PSNR $=27.58 \mathrm{~dB}$

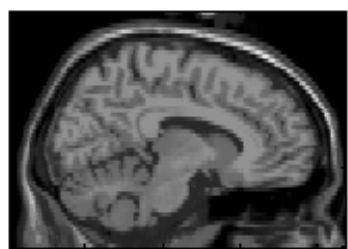

NN PSNR $=23.93 \mathrm{~dB}$

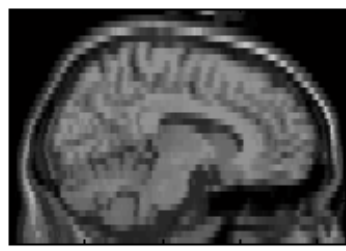

$N N$ PSNR $=22.13 \mathrm{~dB}$

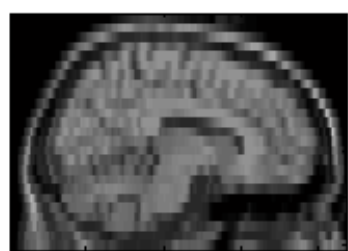

NN PSNR $=20.84 \mathrm{~dB}$

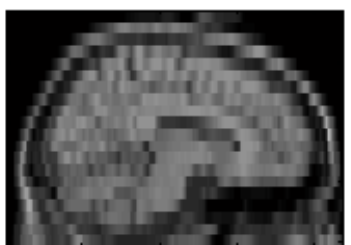

B-Spline PSNR $=37.80 \mathrm{~dB}$

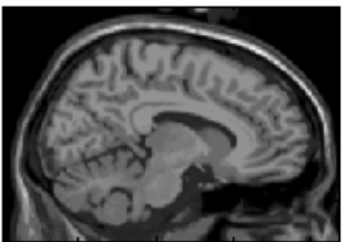

B-Spline PSNR $=32.40 \mathrm{~dB}$

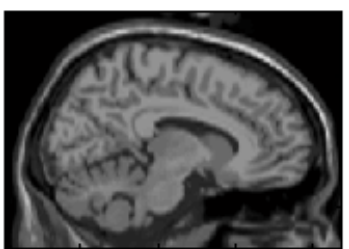

B-Spline PSNR $=26.06 \mathrm{~dB}$

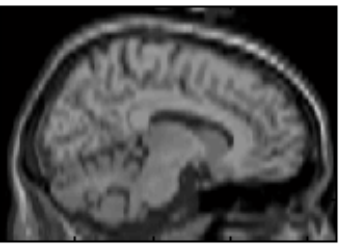

B-Spline PSNR $=23.55 \mathrm{~dB}$

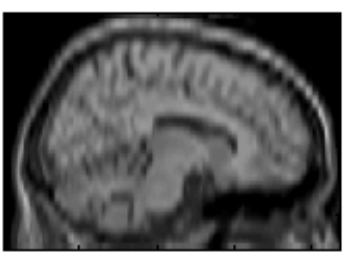

B-Spline PSNR=21.94 dB

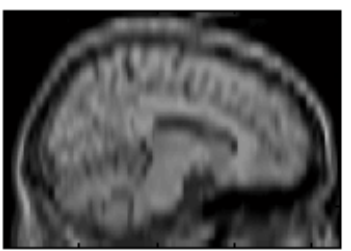

Proposed PSNR $=41.28 \mathrm{~dB}$

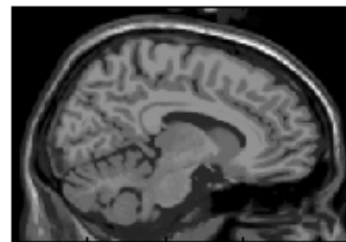

Proposed PSNR $=35.42 \mathrm{~dB}$

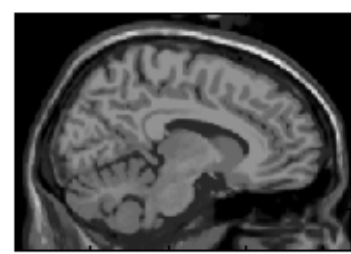

Proposed PSNR $=28.44 \mathrm{~dB}$

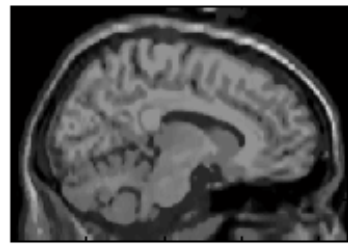

Proposed PSNR $=24.90 \mathrm{~dB}$

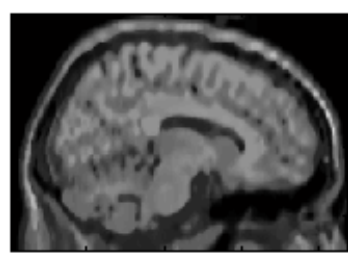

Proposed PSNR=22.66 dB

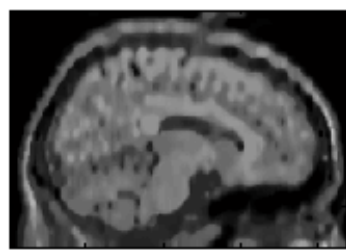

Figure 3. From up to down: Upsampling results of image datasets with different slice thickness $(1,3,5,7$ and $9 \mathrm{~mm})$. From left to right: Original HR data, NN interpolation, B-Spline interpolation and proposed method. As can be noted the proposed approach not only produces the best PSNR in all cases but also the visual appearance seems to be much better than other compared approaches. 
Noisy LR Data
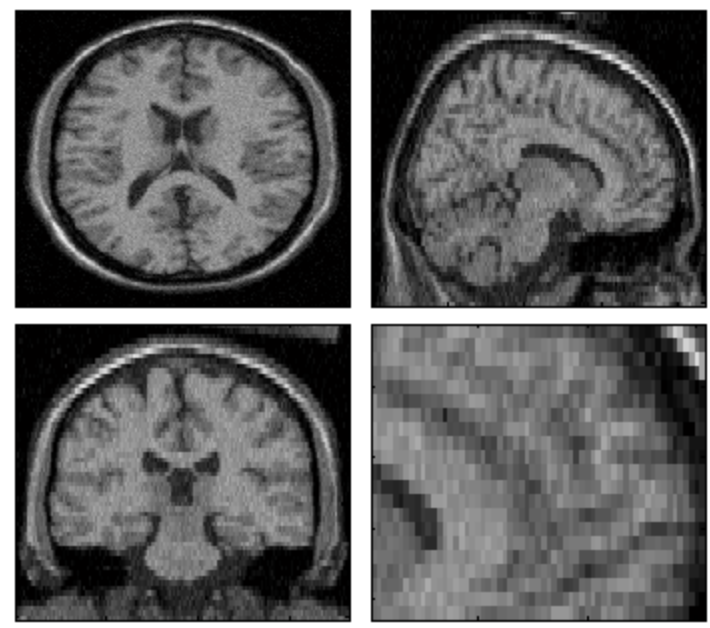

B-Spline interpolation
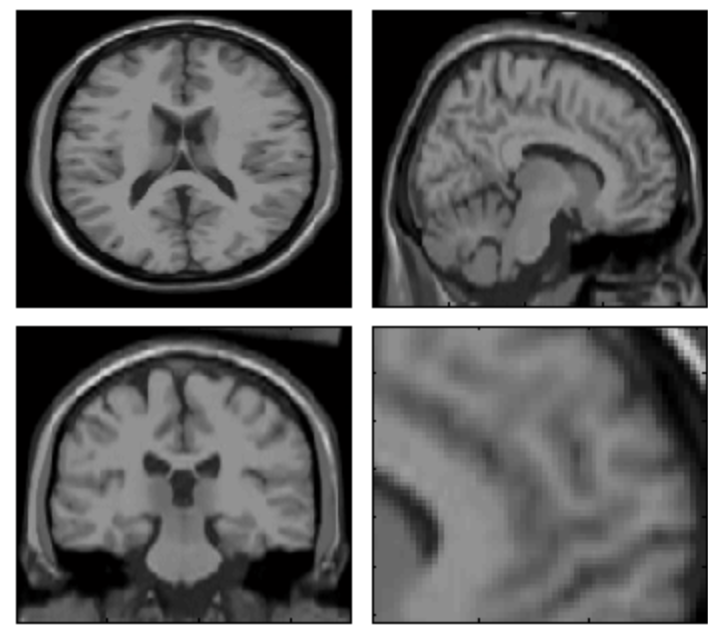

Filtered Data
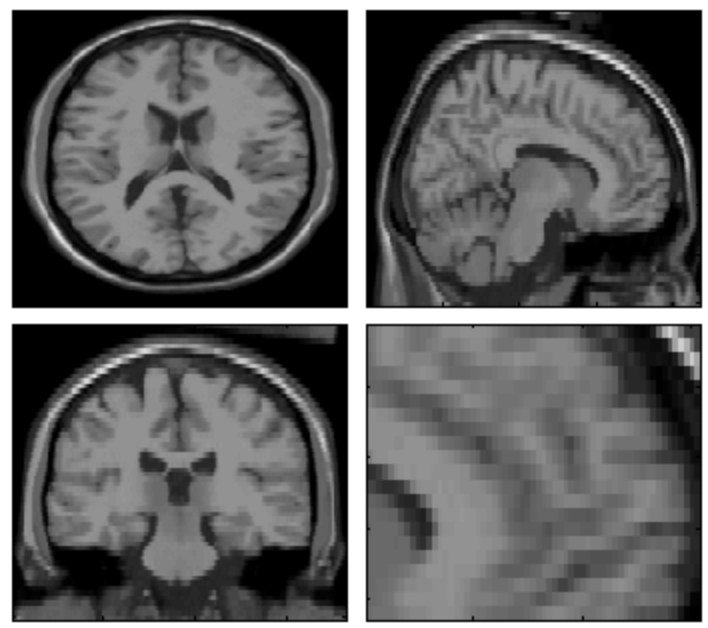

Proposed method
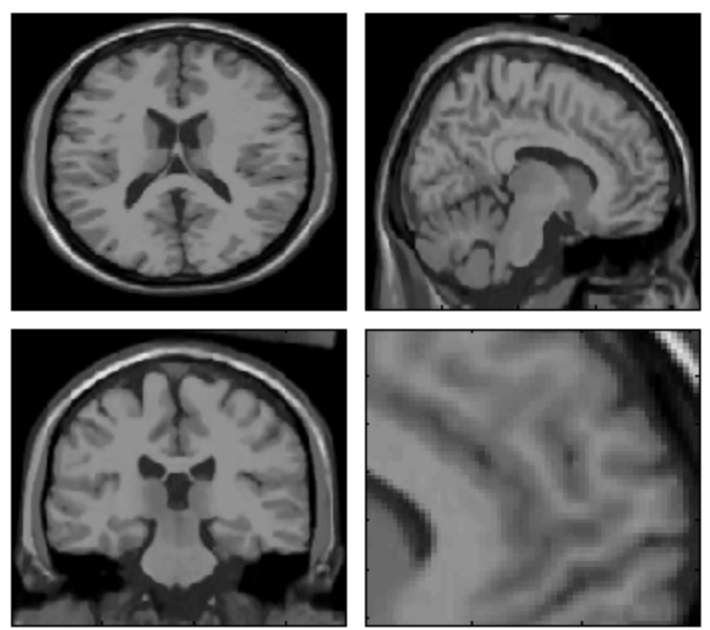

Figure 4. Results of noisy data upsampling. The noisy ( $4 \%$ of Gaussian noise) LR data (voxel resolution $=1 \times 1 \times 3 \mathrm{~mm}^{3}$ ) was upsampled to have a $1 \mathrm{~mm}^{3}$ voxel resolution using the different compared methods. Both B-Spline and the proposed method were applied over the filtered data.

The next experiment consisted in reconstructing an isotropically downsampled versions of the same HR T1-w volume again to $1 \mathrm{~mm}^{3}$ voxel resolution. This HR volume was downsampled to $2 \times 2 \times 2,3 \times 3 \times 3,4 \times 4 \times 4$ and $5 \times 5 \times 5 \mathrm{~mm}^{3}$ and then reconstructed again to $1 \mathrm{~mm}^{3}$. In this experiment no noise was added. The proposed method was again compared to the NN, Trilinear, Cubic and B-Spline $\left(3^{\text {th }}\right.$ order) interpolation methods. The results can be observed on table 3 and figures 5 and 6 . As can be noticed, the proposed method again obtained the best results in all the cases. 
Table 3. PSNR values of the different methods compared for isotropic factors

\begin{tabular}{ccccc}
\hline Factor & $\mathbf{2}$ & $\mathbf{3}$ & $\mathbf{4}$ & $\mathbf{5}$ \\
\hline NN & 26.21 & 22.81 & 20.90 & 19.51 \\
Linear & 28.48 & 24.90 & 22.28 & 20.73 \\
Cubic & 31.33 & 26.46 & 23.55 & 21.70 \\
B-Spline & 32.36 & 27.15 & 23.93 & 21.99 \\
Proposed & $\mathbf{3 5 . 5 0}$ & $\mathbf{2 9 . 6 5}$ & $\mathbf{2 6 . 0 1}$ & $\mathbf{2 3 . 3 9}$ \\
\hline
\end{tabular}

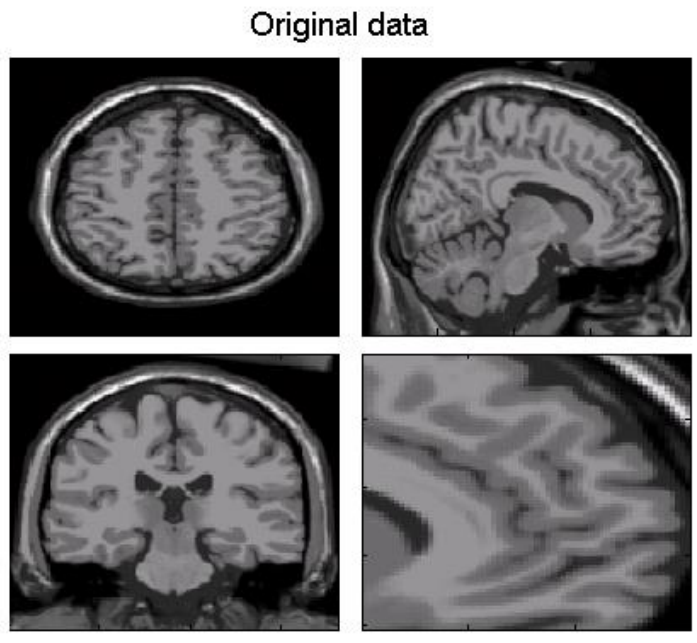

B-Spline
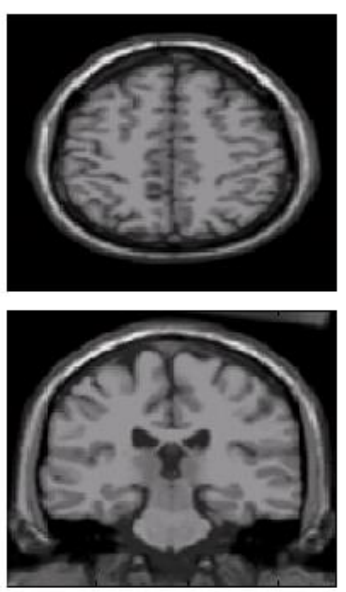
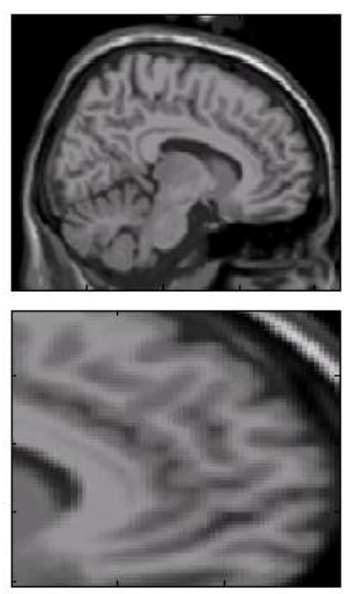

NN
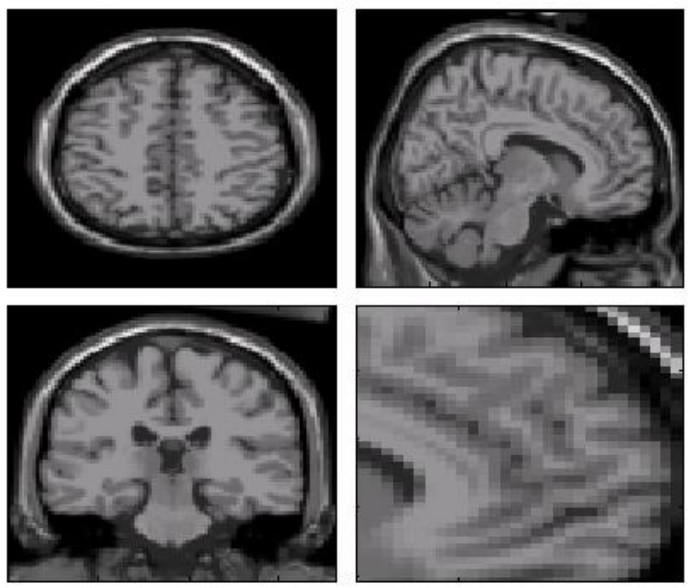

Proposed
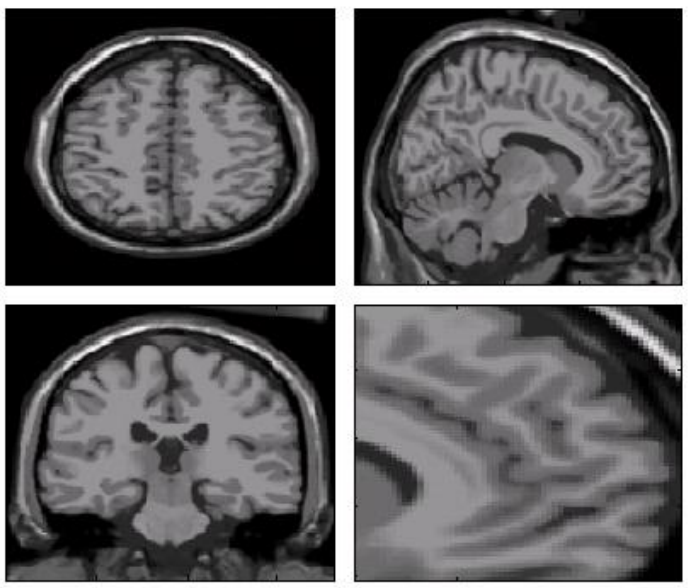

Figure 5. Results of isotropic voxel upsampling. LR data (voxel resolution $=2 \times 2 \times 2 \mathrm{~mm}^{3}$ ) was up sampled to have a $1 \mathrm{~mm}^{3}$ voxel resolution using the different compared methods. It can be noticed that proposed method produces a much compelling result than other compared methods. 
Original data
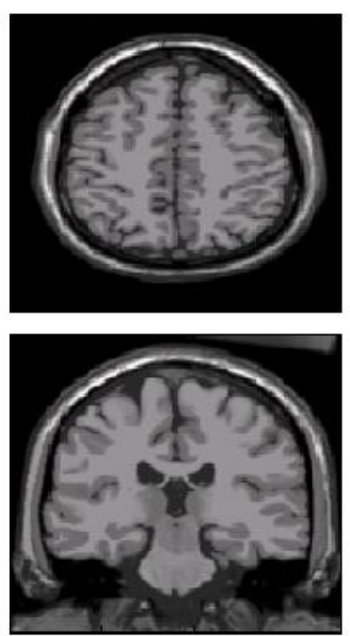

B-Spline
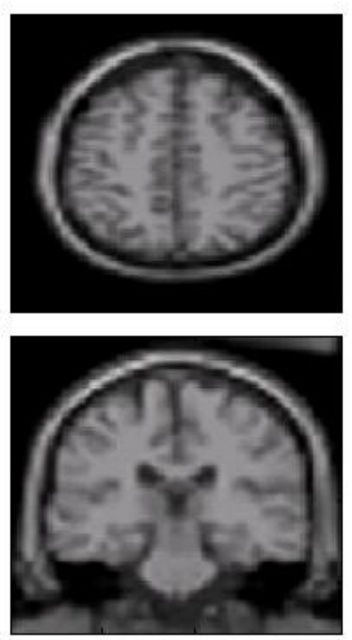
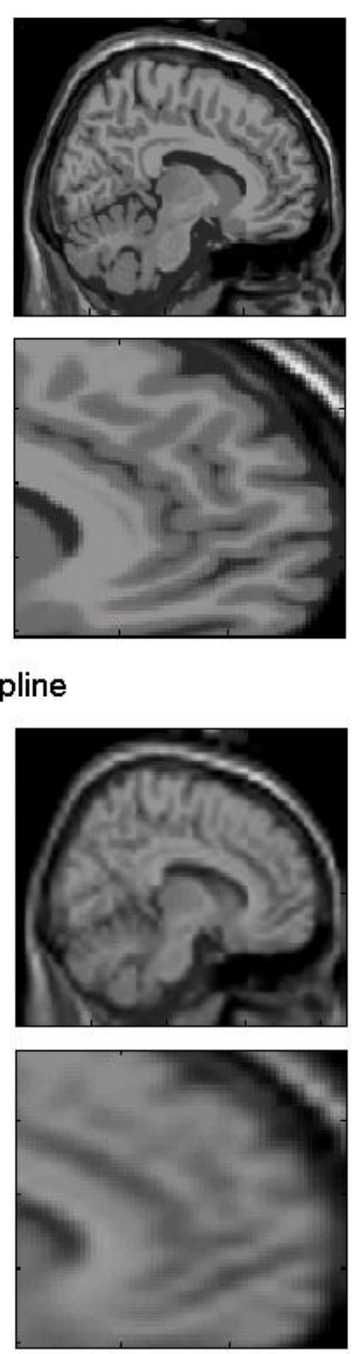

NN
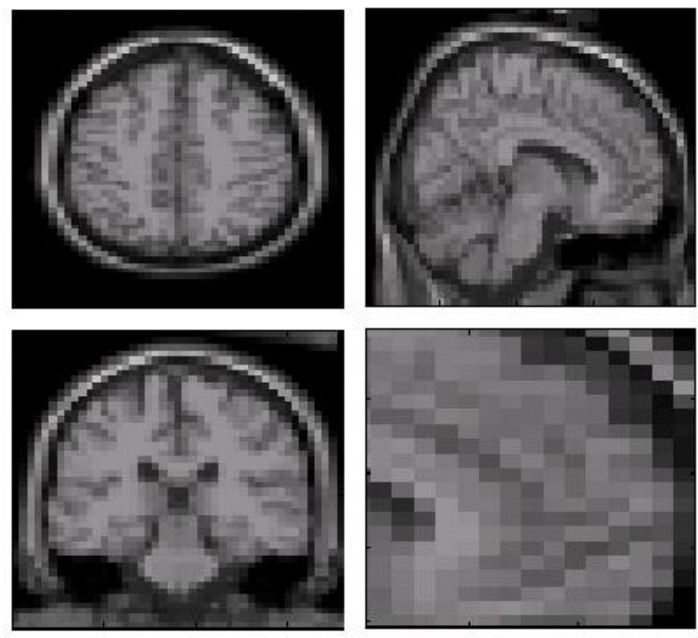

Proposed
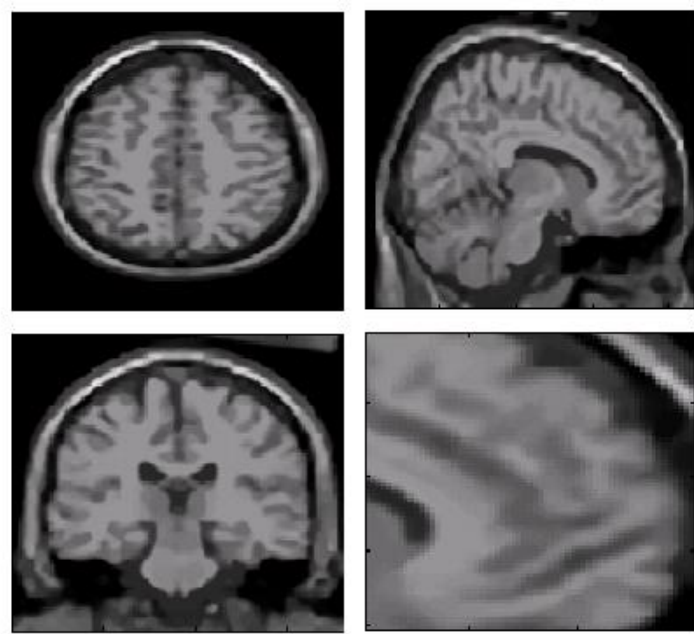

Figure 6. Results of isotropic voxel upsampling. LR data (voxel resolution $=4 \times 4 \times 4 \mathrm{~mm}^{3}$ ) was up sampled to have a $1 \mathrm{~mm}^{3}$ voxel resolution using the different compared methods. It can be noticed that proposed method produces a significative less blurry result than other compared methods.

In order to facilitate reproducibility of the presented experiments the source code of the described experiments and the proposed method can be found at: http://webpage/page.html. 


\subsection{Real clinical data evaluation}

To evaluate the proposed approach on real clinical data, LR and HR T2-w data were used. The LR dataset was obtained using a T2-w volumetric sequence (240x240x53 voxels with a voxels resolution of $1 \times 1 \times 3 \mathrm{~mm}^{3}$ in a Philips Gyroscan 3 Tesla scanner (Netherlands). The HR T2-w data was acquired using the same sequence but using a voxel resolution of $1 \mathrm{~mm}^{3}(240 \times 240 \times 159$ voxels). Both, HR and LR data were filtered using the MNLM3D filter to remove random noise before upsampling.

Two experiments were performed with this data. First a quantitative evaluation of the proposed method was done by upsampling a previously downsampled version of the HR T2-w data $\left(1 \mathrm{~mm}^{3}\right.$ to $\left.1 \times 1 \times 3 \mathrm{~mm}^{3}\right)$. In this way we were able to measure the PSNR of the proposed method $(33.53 \mathrm{~dB})$ and reference B-Spline interpolation (31.22 dB). In the second experiment, the LR T2-w data was upsampled using $B$-spline interpolation and the proposed method and visually compared with the reference HR T2-w data. In figure 7, the results of this qualitative test are shown for visual comparison. As can be noted the proposed method was able to better reconstruct the T2-w data in comparison with BSpline interpolation.

Additionally, the proposed methodology was tested on a pathological dataset containing a brain tumor. In this case, a LR FLAIR-w volume $(224 \times 256 \times 29$ voxels) with a voxel resolution of $0.81 \times 0.81 \times 6 \mathrm{~mm}^{3}$ was used. This dataset (named CEREBRIX) was downloaded from a public MR DICOM data repository (http://pubimage.hcuge.ch:8080/). This LR data was first filtered using the MNLM3D filter and then upsampled to $0.81 \times 0.81 \times 1 \mathrm{~mm}^{3}$ using the B-Spline and the proposed method and the results were qualitatively valuated visually. In figure 8 , the reconstruction results using the compared methods can be observed. It can be easily seen that the upsampled volume using the proposed method is significantly less blurry and better defined than BSpline reconstruction. 
LR data

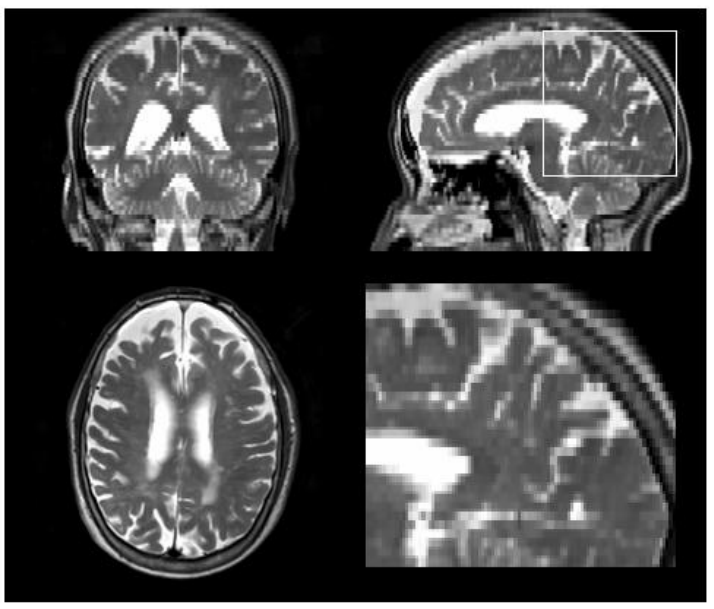

Proposed

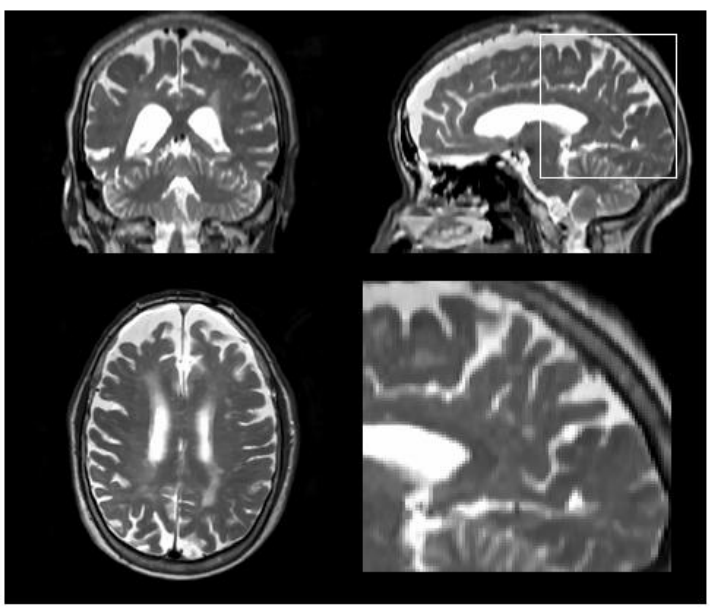

B-Spline interpolation

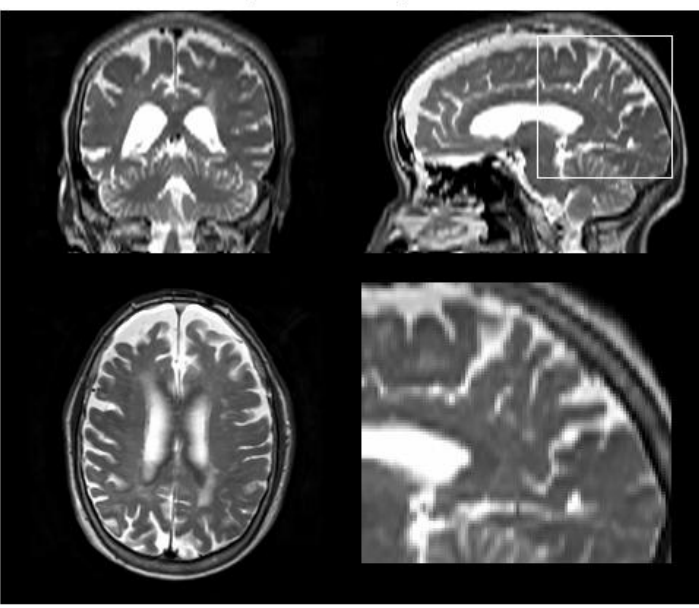

HR reference

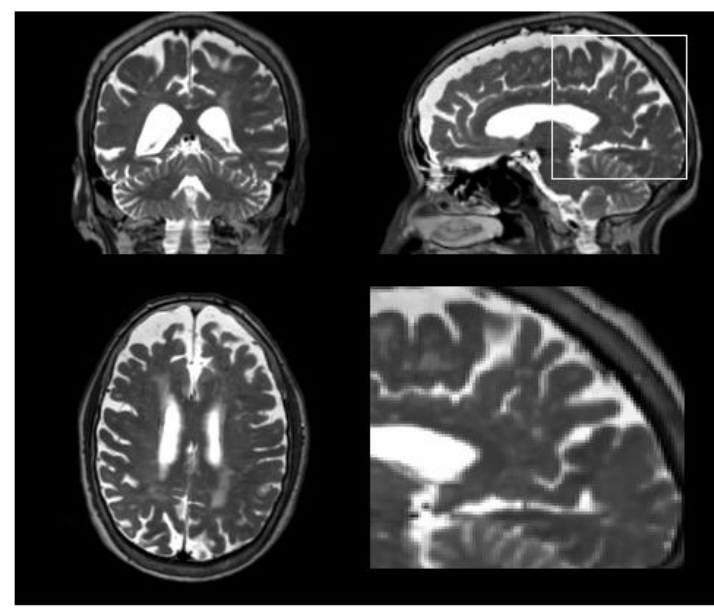

Figure 7. Results of real clinical data upsampling. LR data (voxel resolution $=1 \times 1 \times 3 \mathrm{~mm}^{3}$ ) was upsampled to have a $1 \mathrm{~mm}^{3}$ voxel resolution using the B-Spline and proposed methods. HR reference data is also displayed for visual comparison. Note that dark areas are invented near the ventricles in the axial orientation when using the B-Spline reconstruction. No such areas are visible when using our proposed method. A close up of posterior part of the saggital view is displayed to better show image details. 

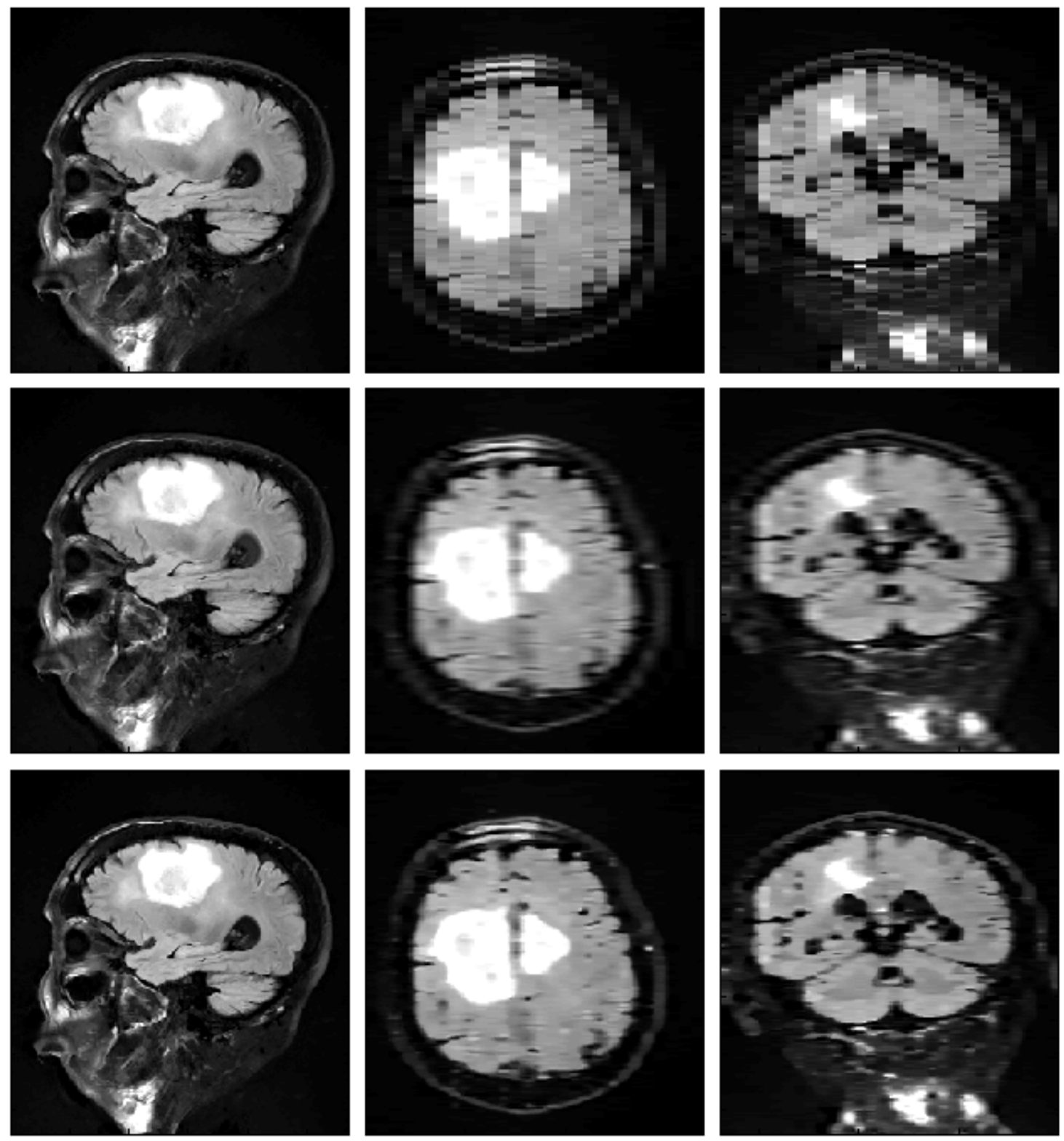

Figure 8. Results of real clinical data upsampling. Highly anisotropic LR data (voxel resolution = $0.81 \times 0.81 \times 6 \mathrm{~mm}^{3}$ ) was up sampled to have a $0.81 \times 0.81 \times 1 \mathrm{~mm}^{3}$ voxel resolution using the BSpline and proposed methods. Top row: Nearest Neighbor interpolation. Middle row: B-Spline interpolation. Bottom row: Proposed method reconstruction. Note that our proposed method produces less blurry images and better defined boundaries. 


\section{Conclusion}

A new method for high quality MR image upsampling has been presented that allows to recover some HR data information from LR data. The proposed method has been demostrated, using synthetic and real data, to outperform classical interpolation methods.

The improved performance of the proposed methodology can be understood under the consideration that the applied regularization has a physically plausible model which has been used to constrain the iterative reconstruction process. Furthermore the regularization performed using the Patch-based non-local scheme prevents the occurrence of the well-known blurring effect typically present in classical interpolation methods by enforcing local image structures.

As the proposed method includes the iterative application of a NLM scheme, its computational burden is high. The typical processing time of a normal MRI volume is around 1 hour (it depends on the number of iterations needed to converge) using a multithreading implementation on quad core normal PC. However, a number of optimizations can be performed to reduce the processing time to few minutes. For example avoiding computations at the background since no useful information is obtained there.

The application of the proposed methodology to upsampled quantitative MRI datasets has to be addressed clinically. Concretely, the application of this technique to upsample DTI studies could be of great interest. This and other possibilities will be addressed with further research. 


\section{Acknowledgements}

The authors want to thank Gracian Garcia-Martí for supplying the MR images used in this paper and for useful discussions about this work. This work has been partially supported by the Spanish Health Institute Carlos III through the RETICS Combiomed, RD07/0067/2001 and by the Canadian grant Industry Cda (CECR)-Gevas-OE016. 


\section{References}

Banerjee J. and Jawahar, C.V. 2008. Super-Resolution of Text Images Using Edge-Directed Tangent Field. The Eighth IAPR International Workshop on Document Analysis Systems, 76-83.

Buades, A., Coll, B., Morel, J.M. 2005. A non local algorithm for image denoising, IEEE Int. Conf. on Computer Vision and Pattern Recognition. 2, 60-65.

Buades A, Coll B, Morel JM, Sbert C., 2009. Self-similarity driven color demosaicking. IEEE Trans Image Process, 18(6):1192-202.

Carmi, E., Liu, S., Alon, N., Fiat, A., Fiat, D. 2006. Resolution enhancement in MRI. Magnetic Resonance Imaging, 24, 133-154.

Cocosco, C.A., Kollokian, V., Kwan, R.K.-S,.Evans, A.C. 1997. BrainWeb: Online Interface to a 3D MRI Simulated Brain Database Proceedings of 3-rd International Conference on Functional Mapping of the Human Brain, 5(4).

Coupé, P., Yger, P., Prima, S., Hellier, P., Kervrann, C., Barillot. C. 2008a. An Optimized Blockwise Non Local Means Denoising Filter for 3D Magnetic Resonance Images. IEEE Transactions on Medical Imaging. 27(4), 425-441.

Coupe, P., Hellier, P., Prima, S., Kervrann, C., Barillot, C. 2008b. 3D Wavelet Subbands Mixing for Image Denoising. International Journal of Biomedical Imaging. Article ID 590183. doi:10.1155/2008/590183.

Criminisi, A., Perez, P., Toyama, K. 2004. Region filling and object removal by exemplar-based image inpainting. IEEE Trans. on Image Proc., 13(9), 12001212. 
Ebrahimi M and Vrscay E. 2007. Solving the inverse problem of image zooming using "self-examples". Lecture Notes in Computer Science. 4633, 117-130.

Efros, A. and Leung, T.K. 1999. Texture synthesis by non-parametric sampling, IEEE International Conference on Computer Vision,1033- 1038.

Elad M. and Datsenko D. 2009. Example-based regularization deployed to super-resolution reconstruction of a single image. The Computer Journal, 52(1):15-30.

Gudbjartsson H. and Patz S. 1995. The Rician distribution of noisy MRI data Magnetic Resonance in Medicine, 34, 910-914.

Kornprobst, P., Peeters, R., Nikolova, M., Deriche, R., Van Hecke M. Ng and P. 2003. A Superresolution Framework for fMRI Sequences and Its Impact on Resulting Activation Maps. MICCAI2003, 2879:117-125.

Lehmann, T. M., Gonner, C. and Spitzer, K. 1999. Survey: Interpolation Methods in Medical Image Processing. IEEE Transaction on Medical imaging, 18(11), 1049-1075.

Manjón, J.V., Carbonell-Caballero, J., Lull, J.J., García-Martí, G., MartíBonmatí, L., Robles, M. 2008. MRI denoising using Non Local Means. Medical Image Analysis, 12(4):514-23.

Nowak R. D. 1999. Wavelet-based Rician noise removal for magnetic resonance imaging. IEEE Transactions on Image Processing, 8(10), 14081419.

Peled, S. and Yeshurun, Y. 2001. Superresolution in MRI: Application to Human White Matter Fiber Tract Visualization by Diffusion Tensor Imaging. Magnetic Resonance in Medicine, 45:29-35. 
Roth, S. Black, S. and M.J. 2005. Fields of experts: A framework for learning image priors. IEEE Conference on Computer Vision and Pattern Recog. (CVPR), 2, 860-867.

Thévenaz, P., Blu, T., Unser, M. 2000. Interpolation Revisited. IEEE Transactions on Medical Imaging, 19(7), 739-758.

Protter M, Elad M, Takeda H, Milanfar P. 2009. Generalizing the NonlocalMeans to Super-Resolution Reconstruction. IEEE Transactions on Image Processing, 18(1), 36-51.

Wiest-Daesslé N, Prima S, Coupé P, Morrissey SP, Barillot C. 2008. Rician noise removal by non-local means filtering for low signal-to-noise ratio mri: Applications to dt-mri. MICCAI2008, 171-179.

Xin Li. 2008. Patch-based image interpolation: algorithms and applications. International Workshop on Local and Non-Local Approximation in Image Processing.

$\mathrm{Xu} \mathrm{H}, \mathrm{Xu} \mathrm{J}$; Wu F. 2008. On the biased estimation of nonlocal means filter. IEEE International Conference on Multimedia.

Zhang W. and Cham, W. 2008. A Single Image Based Blind Superresolution Approach. IEEE International Conference on Image Processing. 12-15. 